\title{
Respect of skin microbiome with different types of leave-on skincare products, an ecobiological approach
}

Sylvie Callejon ${ }^{1}$, Félix Giraud ${ }^{1}$, Florence Larue ${ }^{2}$, Armonie Buisson ${ }^{2}$, Léa Matéos ${ }^{1}$, Laurence Grare $^{1}$, Aurélie Guyoux ${ }^{1}$, Eric Perrier ${ }^{1,3}$, Nathalie Ardiet ${ }^{2}$, and Sandra Trompezinski ${ }^{1}$.

${ }^{1}$ NAOS, Research and Development Department, Aix-en-Provence, France; ${ }^{2}$ NAOS, Cutaneous Investigation and Research Center, Research and Development Department, Lyon, France;

${ }^{3}$ NAOS, Institute of Life Science, Aix-en-Provence, France

\section{Abstract}

Background: Skincare products are used daily to maintain a healthy skin (cleansing, moisturizing, protecting...), but their impact on this first layer, which corresponds to the skin microbiome, is still poorly understood. Preserving natural resources and mechanisms of the skin ecosystem is essential; an original approach based on these premises, called ecobiology, has recently emerged in skincare. Ecobiology considers the skin as an ever-evolving ecosystem which hosts human and microbial cells that interact together with their environment. In this context, we evaluated the impact on the skin microbiome of three types of leave-on face skincare products: a hydrophilic sterile solution, a micellar solution, and an oil-in-water emulsion.

Materials and methods: The individual microbial profiles of 20 Caucasian females were investigated. Samples were obtained twenty-four hours and four days following a daily application of the skincare products versus control area where no product was applied. To analyze the bacterial diversity and abundance of skin microbiome, a 16SrRNA gene sequencing was performed using the Illumina MiSeq platform.

Results: Our results confirm the overall diversity of skin microbiome as previously observed and notably reveal the prevalence of Cutibacterium spp. and Staphylococcus spp. on sebaceous site (the back). Bacterial diversity and abundance were not affected by the products either twenty-four hours or four days after application, as indicated by comparison with the control. Moreover, no dissimilarity was observed between the three products versus their control, neither between each product.

Conclusions: These preliminary results demonstrated for the first time that three different types of leave-on face skincare products such as a hydrophilic sterile solution, a micellar solution, and an emulsion have no impact on skin microbiome and can be considered as "microbiome friendly".

Keywords: skin microbiome, skincare, cosmetic products, ecobiology, microbiome friendly

Corresponding author:

Sandra Trompezinski ${ }^{1}, 355$ rue Pierre Simon Laplace, 13593 Aix-en-Provence Cedex 03, France E-mail address: sandra.trompezinski@,naos.com 


\section{Introduction}

The skin is an ecosystem at the interface with the environment and its primary role is to serve as a physical barrier, cooperating with a chemical defense ( $\mathrm{pH}$, antimicrobial peptides synthesis...) to protect the body from external aggressions. Recent studies revealed that the outer layer of the skin is intimately bound to its microbial communities, the skin microbiome, representing effectively the first line of skin defense; disruptions in its balance can result in skin disorders or infections (Grice \& Segre, 2011). The human skin microbiome hosts fungi, viruses and bacteria, in particular four major phyla: Actinobacteria, Proteobacteria, Firmicutes and Bacteroidetes. The microbiome is essential for the skin equilibrium. Indeed, the cutaneous microbiome protects the skin from pathogens colonization (competition and surface occupation, acidic $\mathrm{pH}$, antimicrobial peptides synthesis), and interacts with the cutaneous immune system in order to educate it tolerating its environment (Chen \& Tsao, 2013). The skin microbiome composition is highly heterogeneous, as it shows intrapersonal variation depending on the localization on the body, the gender, the age, the ethnicity and the environment (climate, stress, diet, life style, drugs, hygiene habits...) (Schommer \& Gallo, 2013; Baldwin et al., 2017; Dimitriu et al., 2019). Concerning the variability depending on the body sites, the microbial composition is influenced by physiological characteristics (Grice et al., 2009; Grice \& Segre, 2011). Three typical broad microenvironment types were identified: sebaceous sites (as the forehead, the face or the back) mainly composed of Propionibacteriaceae including Cutibacterium spp. and Staphylococcaceae; moist sites (as the armpits, the inguinal crease, the popliteal and antecubital fossae) exhibiting a prevalence of Corynebacteriaceae and to a lesser extent of Staphylococcaceae; and dry areas (as the forearm) with greater prevalence of $\beta$ Proteobacteria and Flavobacteria (Grice et al., 2009). Interpersonal variation of the skin microbiome is also observed for a given site (Grice et al., 2009). The biodiversity of the skin microbial ecosystem can be directly linked to the skin's overall health, since skin diseases, like atopic dermatitis and psoriasis, are often associated with dysbiosis (Baldwin et al., 2017). Thus, maintaining an ecological balance of the skin is essential in dermatology; this can be considered as the ultimate aim of ecobiology. Ecobiology is an original approach considering the skin as an ever-evolving ecosystem which hosts human and microbial cells that interact together with their environment and whose natural resources and mechanisms must be preserved (Dréno, 2019; Radman, 2019). This approach applies all the more to skincare products, which play a fundamental role in dermatological treatment for healthy as well as diseased skin. In this context, thanks to the creation of a 3D molecular topographical map to visualize both chemicals and microbial compositions of the skin human surface, Bouslimani et al. succeeded in showing that components of skincare products remained on the skin for weeks after the first use, despite regular showering (Bouslimani et al., 2015, 2019). These components have inevitably an impact on the microbial ecosystem. Actually, Bouslimani 
and colleagues demonstrated an increase in bacterial diversity with deodorant and foot powder after 9 weeks of application, while a little effect for the face sunscreen and the moisturizer on the forearm (Bouslimani et al., 2019). Two other studies reported that deodorants and antiperspirants affected the axillary microbiota by increasing its diversity, and their daily use shifted the microbiome in a stable composition and density by leading to the establishment of a new ecological balance (Callewaert et al., 2014; Urban et al., 2016). Similarly, the use of make-up (foundation and powder) or basic cosmetics (skin softer, lotion, essence, and cream) appeared to increase the microbial diversity on the forehead (Staudinger, Pipal $\&$ Redl, 2011) and on the cheeks (Lee et al., 2018), respectively. As far as skin cleansers are concerned, bacterial communities seemed to reestablish rapidly. Fierer et al. showed an alteration of microbial communities due to hand washing, but the overall levels of bacterial diversity were unrelated to time since last hand washing (Fierer et al., 2008). Moreover, two other studies demonstrated that short-term use of skin cleanser did not significantly affect the cutaneous microbiome in term of diversity and abundance (Numata et al., 2012; Two et al., 2016). Furthermore Perez et al. suggested that the changing of soaps and shampoos may not have substantial impact on the microbial ecology (Perez et al., 2016).

Therefore, despite the increasing awareness on the impact of cosmetic products on skin ecosystem (Holland \& Bojar, 2002; Beri, 2018; Sfriso et al., 2019), few studies have been performed on leave-on skin care products applied daily on the face. The aim of this study is to investigate the impact of three types of leave-on face skin care products (a hydrophilic sterile solution, a micellar solution, and an emulsion) on the skin microbiome diversity and abundance.

\section{Materials \& Methods}

\section{Subject recruitment and sample collection}

Twenty Caucasian subjects between 24 to 46 years (mean age 34.6 years) with phototype II to IV were recruited at the Cutaneous Investigation and Research Center in Lyon (France) during November-December 2018. Participants with the following characteristics were excluded: (1) pregnant or lactating women, (2) smokers, (3) postmenopausal women, (4) began or had a modified hormonal treatment within 3 months, (5) had an auto-immune, severe or progressive disease, (6) had allergy or hypersensitivity to cosmetics, (7) had cutaneous disease on the experimental area, (8) had a sensitive skin, (9) had acne in the back, (10) had diabetes and, (11) had a treatment (antibiotics, anti-inflammatory or anti-acne drugs...) more than 5 consecutive days within the past 4 weeks. Subjects were required to avoid excessive solar exposition during the previous month and swimming pool for a period of 14 days before the study. All subjects signed a written informed consent before participating. All protocols and procedures used in this study were conducted according to the Declaration of Helsinki. The study did not require approval from local ethic committees prior to inclusion of volunteers according to the local legal requirements (Loi Jardé) for non-invasive investigations with cosmetic products on healthy subjects. 
Only women were included in order to rule out differences in the ecosystem of skin microbiome between genders (Fierer et al., 2008; Bouslimani et al., 2019). They were asked to use a generic neutral shampoo and soft cleanser and to otherwise avoid any other product application on the back during 14 days before the study. Three different cosmetic products were applied on the back over an area of $15 \mathrm{~cm}^{2}$ at $1.5 \mu \mathrm{L} / \mathrm{cm}^{2}$ by the same investigator once a day and every day from Day 0 to Day 3. Four areas were defined on the back of each subjects for: (1) a hydrophilic sterile solution (Eau cellulaire mist, Institut Esthederm, NAOS), (2) a micellar solution with surfactants and a preservative (Sensibio H20, Laboratoire Bioderma, NAOS), (3) an oil-in-water emulsion with emulsifiers (B23 Crème fondante hydratante, Etat Pur, NAOS) and, (4) a control without any product application. The swab samples were collected three times: just before the use of the products, on Day 1 and on Day 4. The samples were collected using pre-moistened swabs in sterile-filtered and nuclease free water, BioPerformance Certified (Sigma-Aldrich, St. Quentin Fallavier, France) with the same investigator and following the same swabbing gesture to prevent possible variation across samples. Each swab head was placed in a tube SigmaTranswab® with $1 \mathrm{~mL}$ Liquid Amies medium (Medical Wire \& Equipment Co, Wiltshire, UK) during $30 \mathrm{~min}$ and frozen at $18^{\circ} \mathrm{C}$ until DNA extraction.

\section{DNA extraction from swabs}

The DNA was extracted from the swabs with an optimized and standardized protocol dedicated to bacterial DNA extraction from human samples. An optimized bacterial recovery step from swab was added based on the Nuclospin Microbial DNA kit (Macherey Nagel, France). After DNA extraction, the DNA concentration was increased by PCR.

$\underline{16 S}$ rRNA gene amplification and sequencing

Microbial diversity was determined for each sample by targeted amplification of part of the ribosomal gene. A $16 \mathrm{~S}$ rRNA gene fragment comprising $\mathrm{V} 1$ and V3 hypervariable regions was amplified using an optimized and standardized 16S-ampliconlibrary preparation protocol (Metabiote $\AA$, GenoScreen, Lille, France). Briefly, 16S rRNA gene PCR was carried out using the maximal volume of genomic DNA, according to Metabiote ${ }^{\circledR}$ protocol instructions using 192 bar-coded primers (Metabiote ${ }^{\circledR}$ MiSeq Primers, GenoScreen, Lille, France) at final concentrations of $0.2 \mu \mathrm{M}$ and an annealing temperature of $50^{\circ} \mathrm{C}$ for 30 cycles. PCR products were cleaned up with Agencourt AMPure XP-PCR Purification system (Beckman Coulter, Brea, USA), quantified according to the manufacturer's protocol, and multiplexed at equal concentration. Sequencing was performed using a 300-bp paired-end sequencing protocol on the Illumina MiSeq platform (Illumina, San Diego, USA).

$\underline{\text { Sequence processing and analysis }}$

Raw paired-end reads were subjected to the following process: (1) quality filtering with the PRINSEQ-lite PERL script (Schmieder \& Edwards, 2011) by truncation of bases from the 3 'end not with quality $<30$ based on the Phred algorithm, (2) paired-end read 
assembly using fast length adjustment of short reads (FLASH) to improve genome assemblies (Magoč \& Salzberg, 2011) with a minimum length overlap of 30 bases and $97 \%$ overlap identity and, (3) the search and removal of both forward and reverse primer sequences using CutAdapt, with no mismatches allowed in primers sequences. Assembled sequences for which perfect forward and reverse primers were not found were eliminated. Sequences were clustered into operational taxonomic units (OTUs) with a 97\% identity threshold. Taxonomic assignation was performed using Ribosomal Database Project (RDP) classifier on the Greengenes database V13_8 (DeSantis et al., 2006). A total of 323 volunteers' swabs were collected and used for analysis. Unfortunately 141 samples could not be analyzed because they presented a similar profile than the background in negative controls corresponding to a too low DNA quantity. Since the taxa Escherichia was identified as background in negative controls, a threshold of $15 \%$ of Escherichia reads was applied. The samples with superior relative abundance were discarded from the study, reducing our dataset to 182 samples shared between 8 volunteers at Day 1 and 9 volunteers at Day 4.

\section{$\underline{\text { Data and statistical analysis }}$}

The analysis was performed on the $R$ software. The Shannon-Weaver index, the number of observed OTUs and the BrayCurtis distance matrix were computed with the Phyloseq package (McMurdie and Holmes 2013). The Linear Discriminant Analysis (LDA) effect size (LEfSe) analysis (Segata et al., 2011) was performed on the galaxy web server. Only genera with a LDA score above 3.0 were included. Statistical significance was determined using the Wilcoxon's rank sum test. A p value $<0.05$ was considered as statistically significant.

\section{Results}

16S rRNA gene amplicon sequencing

In total 8,911,437 sequencing reads were generated by sequencing of 323 specimen samples of nineteen volunteers (one volunteer dropped out of the study before completion). Following trimming and quality control, 6,797,231 reads of $16 \mathrm{~S}$ rRNA bacterial sequences were obtained for further analysis. Because biodiversity indexes (Shannon-Weaver index and observed OTUs) are impacted by the number of reads (Morris et al., 2014), samples were normalized at a read depth of 3,100 reads per sample.

$\underline{\alpha \text {-diversity analysis between the three }}$ products

The number of observed OTUs found in a sample is a standard indicator of the diversity. The application of the hydrophilic solution, the micellar solution or the emulsion did not significantly change the number of observed OTUs compared to the control on Day 1 or Day 4 (Fig. 1a). In order to analyze the abundance variations and the number of observed OTUs, the ShannonWeaver index was computed for each sample and compared to the controls. Using the Shannon-Weaver index, no significant differences were found among the hydrophilic solution, the micellar solution and the emulsion compared to their controls (paired Wilcoxon test, $\mathrm{p}>0.05$ ), neither on 
a

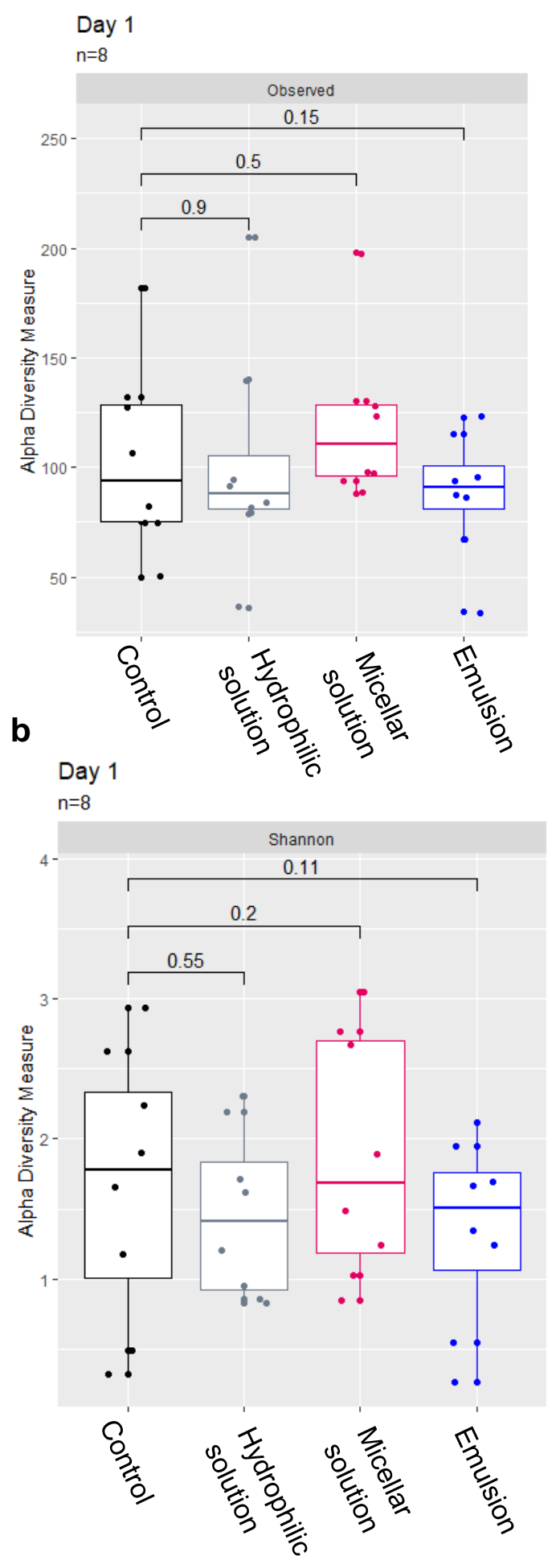

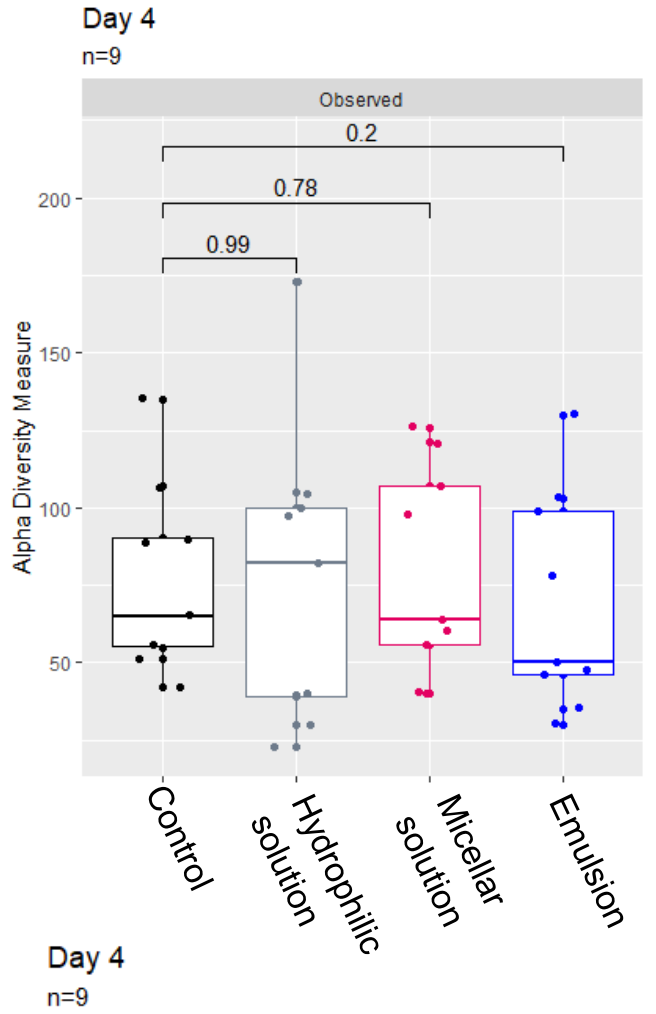

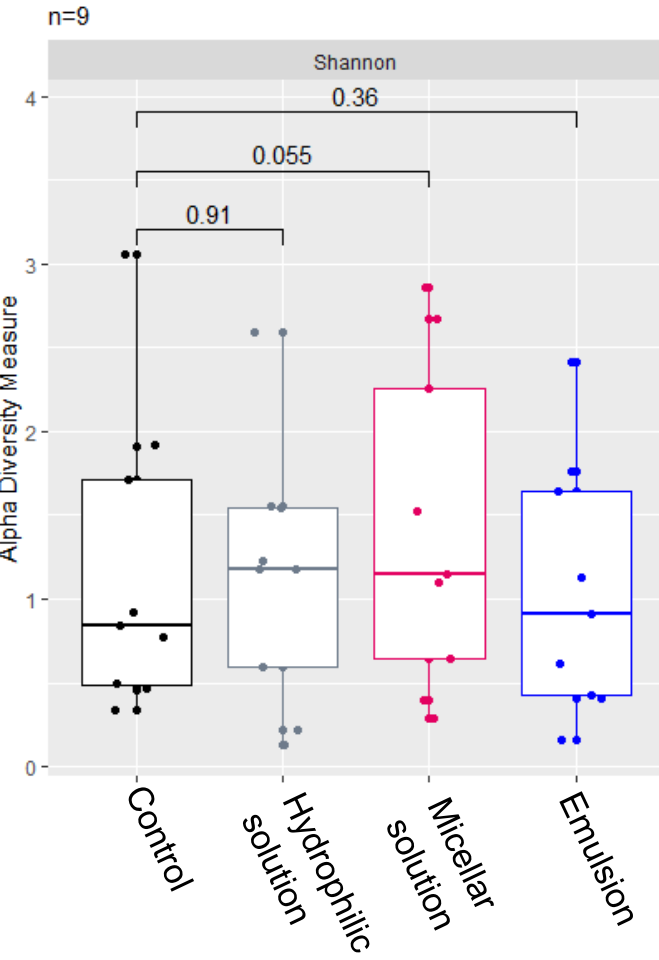

Figure 1: Bacterial diversity at Day 1 and Day 4 using observed OTUs (a) and Shannon-Weaver index (b) of bacterial 16S rRNA gene sequencing reads after 24 hours (Day 1) and 4 days (Day 4) of application of the hydrophilic solution, the micellar solution or the emulsion compared to the control at each time. The p-value was calculated with a paired Wilcoxon test. 
Day 1 nor on Day 4 (Fig. 1b). However, the Shannon-Weaver index is slightly increasing with the application of the micellar solution at Day 4.

Since not enough subjects were in common between Day 1 and Day 4, no statistical comparison was calculated.

Relative abundance analysis between the three products

The relative abundance of the fifteen most abundant genera, representing $94-96 \%$ of the total number of reads (Fig. 2) and at the phylum level (supplementary Fig. S1), demonstrated no major alteration of the skin microbiome after the use of the products compared to their control. The LEfSe, analyzing significant changes at the genus level, demonstrated no significant modification for the hydrophilic solution, the micellar solution and the emulsion on Day 4 (data not shown).

Dissimilarity analysis between the three products

The dissimilarity between each product and the control was investigated using the BrayCurtis distance between each product and their control. The Bray-Curtis distances to the control were then compared between the hydrophilic solution, the micellar solution and the emulsion. No significant differences were observed on Day 1 (Fig. 3a) or Day 4 (Fig. 3b). However, the three products displayed globally low distance compared to their control suggesting that the hydrophilic solution, the micellar solution and the emulsion have a no impact on the skin microbiome.

\section{Discussion}

Firstly, the results of this study confirmed the presence of a high skin microbiome diversity (Fig. 1), associated with Cutibacterium and Staphylococcus as the most dominant genera (Fig. 2), on sebaceous sites, like the back or the face; this is in line with previous observations (Grice et al., 2009). Secondly, the hydrophilic solution, the micellar solution, and the emulsion did not impact negatively the bacterial diversity (Fig. 1) and abundance (Fig. 2); this was observed consistently in a comparison with the control after one application (Day 1) as well as repeated daily application over a period of four days (Day 4). Thus, the three skincare products preserve the skin microbial ecological balance and can be considered as "microbiome friendly" skincare products. Preserving the microbial biodiversity is essential to maintain the natural protection of the skin against pathogens and skin barrier function and to prevent or offset immune system disorders (Baldwin et al., 2017). Indeed it is well established that patients affected by psoriasis or atopic dermatitis have a lower microbial diversity on plaques than on unaffected skin (Byrd et al., 2017; Tett et al., 2017). Maintaining the relative abundance of the most represented taxa is also a challenge for ecobiological skincare products. The application of skincare products can not only alter the skin physiology and consequently the skin microbiome environment, but can also release on the skin new components, which can be beneficial for underrepresented taxa (Lee et al., 2018). Thirdly, no dissimilarity was observed between the products and their control, neither between each product 


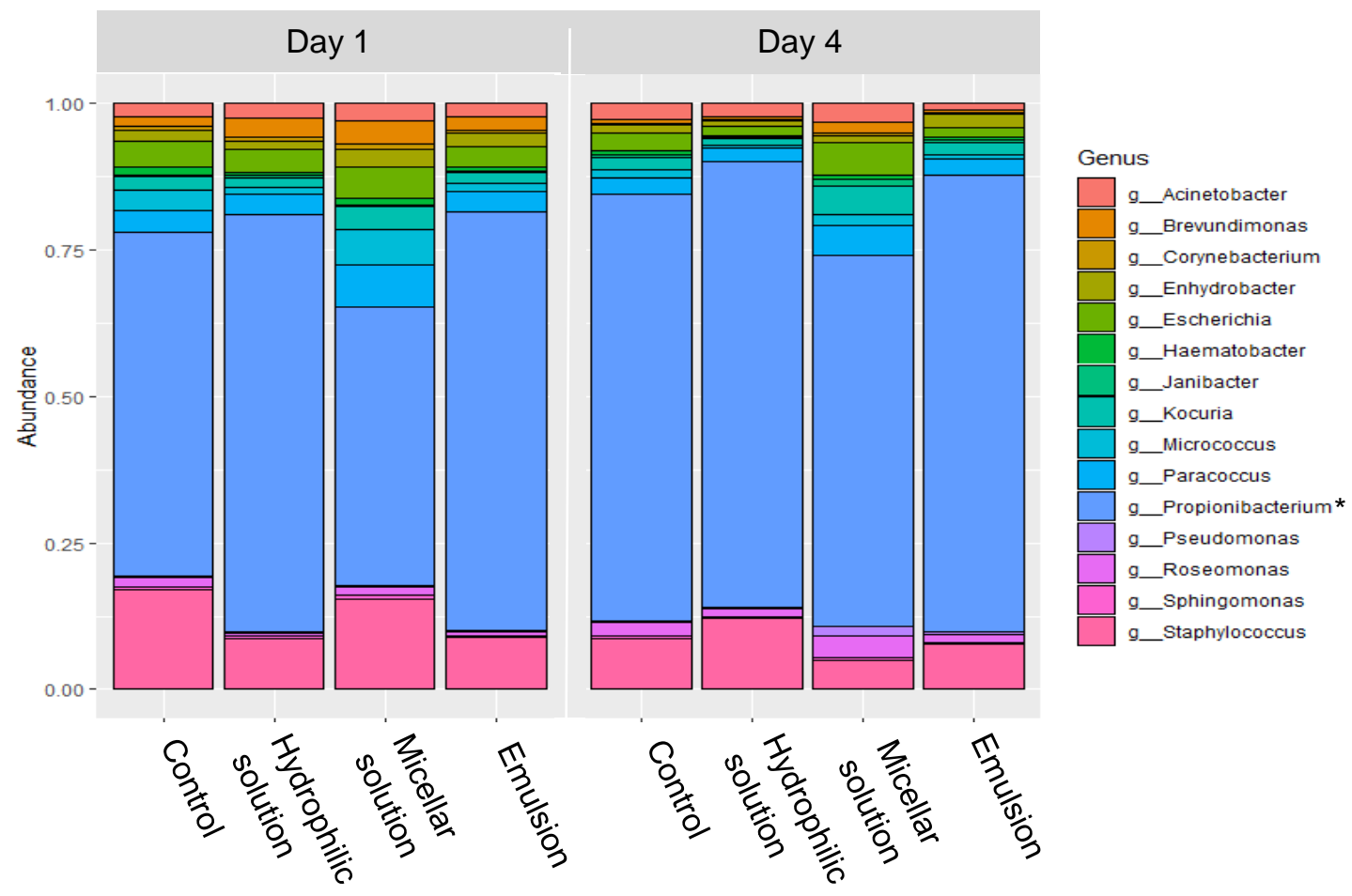

Figure 2: Mean of relative abundance of the 15 most represented bacterial taxas at the genus level after 24 hours (Day 1) and 4 days (Day 4) of application of the hydrophilic solution, the micellar solution or the emulsion compared to their control. See also additional file 1: Fig. S1. *superseded genus name for Cutibacterium
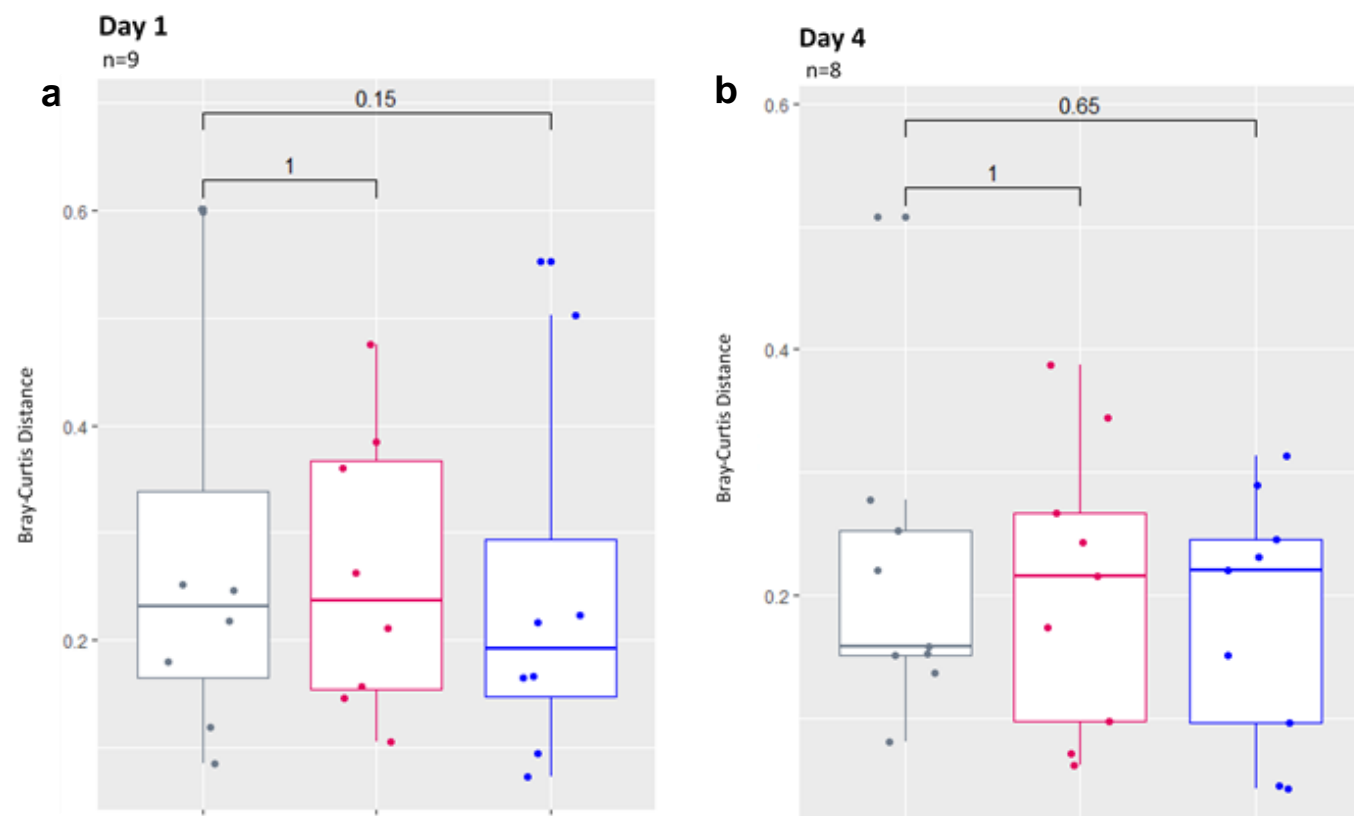

Control vs hydrophyillic solution

Control vs micellar solution

: Control vs emulsion

Figure 3: Bray-Curtis distances after 24 hours (Day 1) (a) and 4 days (Day 4) (b) of application of the hydrophilic solution, the micellar solution or the emulsion versus their control. The p-value was calculated with a paired Wilcoxon test. 
(Fig. 3), despite their different overall composition linked to their function: the micellar solution, a leave-on cleanser with an a surfactant and a preservative, the hydrophilic solution, a sterile sprayable water, and the emulsion, a moisturizing oilin-water emulsion with emulsifiers. Regarding the composition of the products, the hydrophilic solution is a filtered sterile formula at $\mathrm{pH} 6$ filled in an airless aerosol devoid of any preservative ingredient. It presents also bioelectrical characteristics including physiological osmotic pressure. Therefore, we expected that the hydrophilic solution would have no impact on the skin microbiome when applied on the skin. The micellar solution and the emulsion's formulae have a $\mathrm{pH}$ between 4.7 to 5.5 , which simultaneously helps to preserve the formula from microbial contamination and corresponds to physiological skin $\mathrm{pH}$. However, since the formula of these two products is liable to come into contact with air, suitable cosmetic ingredients were added to avoid potential microbial contamination by the consumer's use, as required by cosmetic regulations. Moreover, the micellar solution, as a cleanser, contained also a mild surfactant to solubilize dirt and inevitably, microbes. Yet our results showed no impact on the microbiome profile after the micellar solution nor an emulsion application, confirming the importance of the ingredient choice in term of number (limited ingredients), concentration (in accordance to efficacy and toxicological requirements) and purity (very high quality ingredients, reduced level of potentially harmful trace elements such as heavy metals for instance).
Our study was performed on the back since this area offers a wider surface to apply on a subject the three skincare products and as such, it leaves a suitable spot for a control area; moreover sufficient microbial material for correct DNA amplification can be recovered from the back. In addition, the back benefits from limited exposure to cosmetic products which, as previously mentioned, influence the number and the species of bacteria present on the face. For this reason, even if the investigated cosmetic products are generally applied on the face, we chose to perform sampling on the back, which presents a microbial composition and physiological characteristics comparable to those of the face (Grice \& Segre, 2011). Moreover, it was considered that the back, which is generally less exposed to external factors, may provide a more sensitive tool to evaluate cosmetic products on the skin microbiome. Nevertheless, these preliminary results should be cross-checked by controlled application on the face and also under use conditions, i.e. in the context of twice a day application by the consumer.

As far as we know, this study represents first attempt to assess the impact of leave-on skin cleansers on skin microbiome. Similarly to our results on the micellar solution on Day 1, comparable studies evaluating rinsed skin cleansers showed that bacterial communities seemed to regain rapidly their diversity and abundance following product application (Fierer et al., 2008; Numata et al., 2012; Two et al., 2016). Since the micellar solution is left on, like the hydrophilic solution and the emulsion, we also evaluated the skin microbiome on Day 4, and no significant changes on bacterial diversity and abundance 
were observed (Fig. 1 and 2). As far as the hydrophilic solution and the emulsion are concerned, Lee et al. demonstrated on thirty Korean females that the application twice a day of basic cosmetics consisting of a skin softer (solubilized type), a lotion (oil in water), an essence (solubilized type), and a cream (oil-in-water emulsion type) during four weeks appeared to increase microbial diversity on the cheeks (Lee et al., 2018). Therefore, the use of these basic cosmetic products seemed to negatively impact the skin microbiome's equilibrium. The discordant results observed in our study could be partially explained by considering the different experimental conditions tested and the ingredients quality and the simplicity of the formula. Lee et al. performed their experiments on the cheeks, by analyzing a larger sample group and by performing a longer application in use conditions. Moreover, several cosmetic products were applied sequentially. To the best of our knowledge, only one study was performed with a moisturizing emulsion. This analysis demonstrated a moderate increase in bacterial diversity after a daily application of the emulsion on front forearms of twelve subjects over a period of three weeks (Bouslimani et al., 2019). By contrast, we observed no changes on bacterial diversity and abundance after daily application of the emulsion over a period of 4 days (Fig. 1 and 2). These preliminary results must therefore be confirmed by studying a daily application over a longer timeframe and by increasing the sample size to evaluate temporal diversity. This study was limited to the 16S rRNA sequencing and bacterial identification. In order to deepen and refine the analysis, further investigations should explore microbiome variations after the application of cosmetic products using shotgun sequencing. In conclusion, these preliminary results show that a hydrophilic solution, a micellar solution, and an emulsion, all formulated with selected cosmetic ingredients according to the ecobiological approach, do not alter significantly the skin microbiome's balance over several days of application. This study lays the foundations for a new way of formulating skincare products, according to which the unique skin microbiome profile of each individual should be determined in order to cater for individual skin needs. Indeed our ecobiological values demonstrated us day after day that, more than a barrier against external aggressions, the skin is an interface between two alive worlds.

\section{Acknowledgements}

Drafting of the article was assisted by Marlène Chavagnac-Bonneville and Jacopo Novelli, NAOS, France.

\section{Conflicts of Interest}

This study was funded by NAOS, France. Sylvie Callejon, Félix Giraud, Florence Larue, Armonie Buisson, Léa Matéos, Laurence Grare, Aurélie Guyoux, Eric Perrier, Nathalie Ardiet, and Sandra Trompezinski are employees of NAOS, France.

\section{References}

Baldwin HE, Bhatia ND, Friedman A, Martin R, Seité S. 2017. The role of cutaneous microbiota harmony in maintaining a functional skin barrier. Journal of Drugs in Dermatology. DOI: 
10.25251/skin.1.supp.138.

Beri K. 2018. Skin microbiome \& host immunity: Applications in regenerative cosmetics \& transdermal drug delivery. Future Science OA. DOI: 10.4155/fsoa2017-0117.

Bouslimani A, Porto C, Rath CM, Wang M, Guo Y, Gonzalez A, Berg-Lyon D, Ackermann G, Christensen GJM, Nakatsuji T, Zhang L, Borkowski AW, Meehan MJ, Dorrestein K, Gallo RL, Bandeira N, Knight R, Alexandrov T, Dorrestein PC. 2015. Molecular cartography of the human skin surface in 3D. Proceedings of the National Academy of Sciences of the United States of America. DOI: 10.1073/pnas.1424409112.

Bouslimani A, Da Silva R, Kosciolek T, Janssen S, Callewaert C, Amir A, Dorrestein K, Melnik A V., Zaramela LS, Kim JN, Humphrey G, Schwartz T, Sanders K, Brennan C, Luzzatto-Knaan T, Ackermann G, McDonald D, Zengler K, Knight R, Dorrestein PC. 2019. The impact of skin care products on skin chemistry and microbiome dynamics. BMC Biology. DOI: 10.1186/s12915019-0660-6.

Byrd AL, Deming C, Cassidy SKB, Harrison OJ, Ng WI, Conlan S, Belkaid Y, Segre JA, Kong HH. 2017. Staphylococcus aureus and Staphylococcus epidermidis strain diversity underlying pediatric atopic dermatitis. Science Translational Medicine 9.

DOI: 10.1126/scitranslmed.aal4651.

Callewaert C, Hutapea P, Van de Wiele T, Boon N. 2014. Deodorants and antiperspirants affect the axillary bacterial community. Archives of Dermatological Research. DOI: 10.1007/s00403-014-1487-1.

Chen YE, Tsao H. 2013. The skin microbiome: Current perspectives and future challenges. Journal of the
American Academy of Dermatology. DOI: 10.1016/j.jaad.2013.01.016.

DeSantis TZ, Hugenholtz P, Larsen N, Rojas M, Brodie EL, Keller K, Huber T, Dalevi D, Hu P, Andersen GL. 2006. Greengenes, a chimera-checked 16S rRNA gene database and workbench compatible with ARB. Applied and Environmental Microbiology 72:50695072. DOI: 10.1128/AEM.03006-05.

Dimitriu PA, Iker B, Malik K, Leung H, Mohn WW, Hillebrand GG. 2019. New insights into the intrinsic and extrinsic factors that shape the human skin microbiome. mBio. DOI: 10.1128/mBio.00839-19.

Dréno B. 2019. The microbiome, a new target for ecobiology in dermatology. European Journal of Dermatology. DOI: $10.1684 /$ ejd.2019.3535.

Fierer N, Hamady M, Lauber CL, Knight R. 2008. The influence of sex, handedness, and washing on the diversity of hand surface bacteria. Proceedings of the National Academy of Sciences of the United States of America. DOI: 10.1073/pnas.0807920105.

Grice EA, Kong HH, Conlan S, Deming CB, Davis J, Young AC, Bouffard GG, Blakesley RW, Murray PR, Green ED, Turner ML, Segre JA. 2009. Topographical and temporal diversity of the human skin microbiome. Science. DOI: $10.1126 /$ science. 1171700 .

Grice EA, Segre JA. 2011. The skin microbiome. Nature Reviews Microbiology.

DOI: 10.1038/nrmicro2537.

Holland KT, Bojar RA. 2002. Cosmetics: What is their influence on the skin microflora? American Journal of Clinical Dermatology. DOI: 10.2165/00128071-200203070-00001.

Lee HJ, Jeong SE, Lee S, Kim S, Han H, Jeon CO. 2018. Effects of cosmetics on the skin microbiome of facial cheeks with 
different hydration levels. MicrobiologyOpen. DOI: 10.1002/mbo3.557.

Magoč T, Salzberg SL. 2011. FLASH: Fast length adjustment of short reads to improve genome assemblies. Bioinformatics 27:2957-2963. DOI: 10.1093/bioinformatics/btr507.

Morris EK, Caruso T, Buscot F, Fischer M, Hancock C, Maier TS, Meiners T, Müller C, Obermaier E, Prati D, Socher SA, Sonnemann I, Wäschke N, Wubet T, Wurst S, Rillig MC. 2014. Choosing and using diversity indices: insights for ecological applications from the German Biodiversity Exploratories. Ecology and Evolution 4:3514-3524. DOI: $10.1002 /$ ece 3.1155.

Numata S, Akamatsu H, Akaza N, Sasaki Y, Takeoka S, Mizutani H, Nakata S, Matsunaga K. 2012. Quantitative effect of face washing on cutaneous resident microbiota in female subjects who wear make-up. Journal of Dermatology. DOI: 10.1111/j.1346-8138.2012.01644.x.

Oh J, Byrd AL, Park M, Kong HH, Segre JA. 2016. Temporal Stability of the Human Skin Microbiome. Cell. DOI: 10.1016/j.cell.2016.04.008.

Perez GIP, Gao Z, Jourdain R, Ramirez J, Gany F, Clavaud C, Demaude J, Breton L, Blaser MJ. 2016. Body site is a more determinant factor than human population diversity in the healthy skin microbiome. PLOS ONE. DOI: 10.1371/journal.pone.0151990.

Radman M. 2019. Ecobiological approach to research regarding ageing and diseases. European Journal of Dermatology. DOI: 10.1684/ejd.2019.3534.

Schmieder R, Edwards R. 2011. Quality control and preprocessing of metagenomic datasets. Bioinformatics 27:863-864. DOI:

10.1093/bioinformatics/btr026.

Schommer NN, Gallo RL. 2013. Structure and function of the human skin microbiome. Trends in Microbiology. DOI: 10.1016/j.tim.2013.10.001.

Sfriso R, Egert M, Gempeler M, Voegeli R, Campiche R. 2019. Revealing the secret life of skin with the microbiome you never walk alone. International Journal of Cosmetic Science. DOI: 10.1111/ics.12594.

Staudinger T, Pipal A, Redl B. 2011. Molecular analysis of the prevalent microbiota of human male and female forehead skin compared to forearm skin and the influence of make-up. Journal of Applied Microbiology. DOI: 10.1111/j.1365-2672.2011.04991.x.

Tett A, Pasolli E, Farina S, Truong DT, Asnicar F, Zolfo M, Beghini F, Armanini F, Jousson O, De Sanctis V, Bertorelli R, Girolomoni G, Cristofolini M, Segata N. 2017. Unexplored diversity and strain-level structure of the skin microbiome associated with psoriasis. npj Biofilms and Microbiomes 3:1-12. DOI: 10.1038/s41522-0170022-5.

Two AM, Nakatsuji T, Kotol PF, Arvanitidou E, Du-Thumm L, Hata TR, Gallo RL. 2016. The Cutaneous Microbiome and Aspects of Skin Antimicrobial Defense System Resist Acute Treatment with Topical Skin Cleansers. Journal of Investigative Dermatology. DOI: 10.1016/j.jid.2016.06.612.

Urban J, Fergus DJ, Savage AM, Ehlers M, Menninger HL, Dunn RR, Horvath JE. 2016. The effect of habitual and experimental antiperspirant and deodorant product use on the armpit microbiome. PeerJ. DOI: 10.7717/peerj. 1605 . 